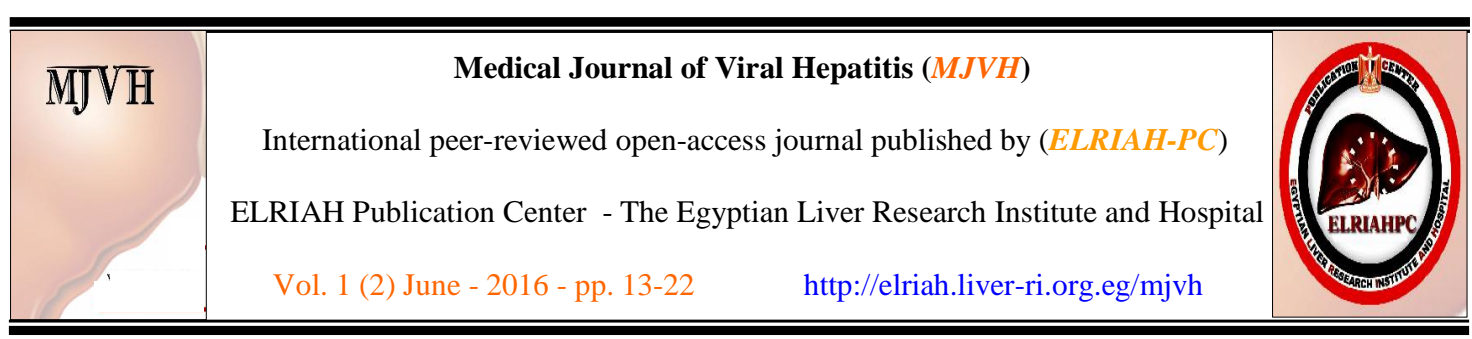

Original article

\title{
A simple bedside blood test (Fibrofast; FIB-5) is superior to FIB-4 index for the differentiation between non-severe and severe fibrosis in patients with chronic Hepatitis C
}

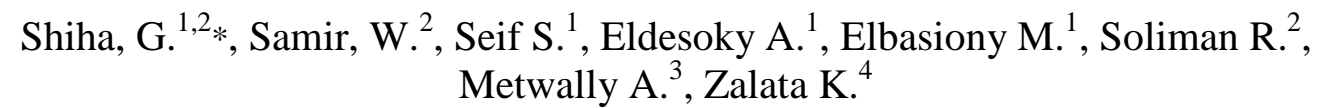

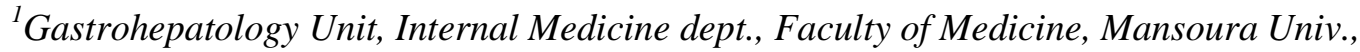
Egypt.

${ }^{2}$ Egyptian Liver Research Institute and Hospital (ELRIAH), Mansoura, Egypt.

${ }^{3}$ Community Medicine Department, National Research Center, Cairo, Egypt

${ }^{4}$ Pathology Department, Faculty of Medicine, Mansoura University, Egypt.

* E-mail: g_shiha@hotmail.com

Article History

Received: 2/3/2016

Revised: 30/7/2016

Accepted: 1/9/2016

\section{Keywords:}

Fibrofast (FIB-5)

FIB-4

Liver fibrosis

Liver biopsy

Hepatitis C Genotype 4

\begin{abstract}
:
A simple noninvasive score (Fibrofast, FIB-5) was developed using five routine laboratory tests (ALT, AST, Alkaline phosphatase, Albumin and Platelets count) for the detection of severe hepatic fibrosis in patients with chronic hepatitis $C$. The FIB-4 index is a noninvasive test for the assessment of liver fibrosis, and a score of $\leq 3.35$ enables the correct identification of patients who have nonsevere (FO-2) from severe fibrosis (F3 4), and could avoid liver biopsy. The aim of this study was to compare the performance characteristics of FIB-5 and FIB-4 to differentiate between nonsevere from severe fibrosis. A cross-sectional study included 604 chronic HCV patients. All liver biopsies were scored using METAVIR system. Both FIB-5 and FIB-4 scores were measured and the performance characteristics were calculated using the ROC curve. The performance characteristics of Fibro-Fast at $\geq-2.1$ and FIB-4 at $\leq 3.25$ for the differentiation between non-severe fibrosis and severe fibrosis were; sensitivity $39.6 \%, N P V \quad 88.7 \%$ and sensitivity $29.7 \%$, NPV $87.4 \%$ respectively. Conclusion: FIB-5 score at the new cutoff is more superior to FIB-4 index for the differentiation between non- severe and severe fibrosis.
\end{abstract}

Abbreviations: ALP; alkaline phosphatase. ALT; alanine aminotransferase. AST; aspartate aminotransferase. $\mathrm{CHC}$; chronic hepatitis $\mathrm{C} . \mathrm{HCV}$; hepatitis $\mathrm{C}$ virus. NPV: negative predictive value. PPV: positive predictive value, $S D$; standard deviation. Sn: sensitivity, SP: specificity. 


\section{Introduction}

Assessment of liver fibrosis in patients with chronic hepatitis $\mathrm{C}$ virus (HCV) infection is considered a relevant part of patient care, for decision making, and is an important prognostic factor [1,2]. In addition, the severity of liver fibrosis may be used as a selection criterion for antiviral therapy, duration of treatment, surveillance for hepatocellular carcinoma (HCC), and for esophageal varices screening [3,4]. The gold standard for assessing the health of the liver is the liver biopsy. In addition to histological evaluation, liver biopsy also provides information on necroinflammatory activity and other features, such as steatosis and hepatic deposits of iron or copper. Besides its advantages, liver biopsy is an invasive technique with associated morbidity, mortality, and complications [5]. Other problems associated with the use of liver biopsy in assessing fibrosis staging are related to sample size and the heterogeneity of pathology in chronic hepatitis $\mathrm{C}$ (CHC) infection, and whether this sample is $100 \%$ representative of the entire liver [6-12]. Previous reports have proposed that a liver biopsy sample should contain a minimum of 5 portal tracts and be at least $15 \mathrm{~mm}$ in length to be considered adequate [8,13-14]. Others have recommended core length $>15$ $\mathrm{mm}$ or contain at least 10 portal tracts $[15,16]$, but this might need more than one path with the biopsy needle to be achieved. Considering these limitations, many studies have recently focused on the development of non-invasive markers as an attractive surrogates of liver biopsy for both patients and physicians [17-27]. Besides the clear advantage of being noninvasive, may overcome the mentioned intra- and interobserver variability of liver biopsy, theoretically these tests offer a more accurate view of fibrogenic events occurring in the entire liver and carry the advantage of providing frequent fibrosis evaluation without additional risk. Fibrofast (FIB-5) and the FIB-4 are a simple noninvasive markers that score for liver fibrosis evaluated on the basis of simple biomarkers (ALT:AST ratio, albumin, alkaline phosphatase, and platelets count) and simple variables such as age, AST, ALT, and platelet count respectively, and can be used by the clinicians to predict severe fibrosis or cirrhosis in patients with CHC infection [27,28]. The objective of this study in Egyptian patients with $\mathrm{CHC}$ was to compare the performance characteristics of FIB-5 and FIB-4 to differentiate between non-severe (F0-2) from severe fibrosis (F3-4).

\section{Patients and Methods}

\subsection{Patients}

This cross-sectional study conducted on 604 patients chronically infected with $\mathrm{HCV}$ genotype 4 (HCV-G4) attending the Egyptian Liver hospital, Mansoura, Egypt in the period from 2013 to 2015. HCV RNA positive patients were identified among HCV antibody (anti-HCV Ab) positive patients. Later, the study plan was discussed with patients and the biopsy was taken only from those patients who were willing for this procedure.

\subsection{Patients' Consent}

Informed written consent from each patient and local ethical committee 
approval were obtained before starting the data collection. With respect to patients' confidentiality, patients were represented in the study by code numbers. All personal data was concealed. The study protocol conformed to the ethical guidelines of the 1975 Declaration of Helsinki as reflected in a priori approval by the institution's human research committee.

\subsection{Exclusion Criteria}

Patients who have received any previous courses of antiviral or immunosuppressive therapy, those who had clinical evidence of HBV or HIV infection, and those with any type of liver cancer were excluded from the study. Patients who refused to have a liver biopsy or for whom it was contraindicated, i.e., because of a low platelet count, prolonged prothrombin time or decompensated cirrhosis were also excluded from the study.

\subsection{Baseline assessment:}

The patients were subjected to thorough history taking, clinical examination, routine laboratory work-up including complete blood count, international normalized ratio (INR), and serum aspartate aminotransferase (AST), alanine aminotransferase (ALT), alkaline phosphatase (ALP), albumin, and bilirubin.

\subsection{Calculations}

The FIB-5 score was calculated according to Attallah et al., 2006 [28] as follow:

\section{albumin $(\mathrm{g} / \mathrm{L}) \times \mathbf{0 . 3}+$ platelet count $\left(10^{9} / \mathrm{L}\right) \times 0.05-$ alkaline phosphatase $($ IU/L $) \times 0.014$ + AST/ALT ratio $\times 6$ + 14 .}

The FIB-4 [30] score was calculated as follows:

\author{
[Age (year) $\times$ AST (IU/L)] / [platelet \\ count $\left(\times 10^{9} / \mathrm{L}\right) \times$ ALT $\left.(\text { IU/L })^{1 / 2}\right]$.
}

\subsection{HCV RNA detection and quantitative PCR \\ RNA was extracted from $140 \mu \mathrm{l}$} serum samples using QIAamp viral RNA extraction kit (Qiagen USA cat \# 52906) according to the manufacturer's protocol. cDNA was synthesized using Moloney murine leukemia virus (MmLV) reverse transcriptase (Invitrogen, USA). First round and nested PCRs were carried out with Taq Polymerase (Fermentas USA) and analyzed on $2 \%$ agarose gel. Qiagen $\mathrm{HCV}$ quantitative kit was used to perform $\mathrm{HCV}$ RNA quantification amplification after each replicating cycle with $10 \mu$ of the extracted RNA on Roche Real Time PCR using fluorescent probes to detect.

\subsection{HCV genotyping}

HCV genotyping was carried out using Invader $\mathrm{HCV}$ genotyping assay (Third wave technology, USA). Briefly, about 100 ng of the HCV RNA was reverse transcribed to cDNA using $200 \mathrm{U}$ of MmLV (Invitrogen, USA). From the amplified product, $2 \mu \mathrm{l}$ was taken and the genotyping assay was performed for 12 different $\mathrm{HCV}$ types.

\subsection{Liver Biopsy and Histo-pathological examination}

The liver biopsy procedure, its advantages, and its possible adverse effects were explained to the patients. An informed consent to obtain a liver biopsy was obtained from all patients. Histopathological examination of ultrasound guided percutaneous liver biopsy requires using 16 gauge semiautomated biopsy needles. Liver specimens of $15 \mathrm{~mm}$ in length with a 
minimum of 4 portal tracts were fixed in $10 \%$ neutral formalin, then processed and embedded in paraffin. Sections were stained with Hematoxylin and Eosin, and Masson trichrome stains for detection of fibrosis. METAVIR scoring system demonstrated different stages of fibrosis (F0-4) [29]. The histopathological examination of all liver biopsies was performed by a single hepatology expert pathologist. Histological staging based on the degree of fibrosis have five degrees of fibrosis: as F0 (no fibrosis), F1 (mild fibrosis without septa), F2 (moderate fibrosis with few septa), F3 (severe fibrosis with numerous septa without cirrhosis) and F4 (cirrhosis). We further grouped fibrosis stages into two groups; the first as F0-2 (non-severe fibrosis), and the second as F3-4 (severe fibrosis).

\subsection{Statistical Analysis}

Data were collected in a preformed Data Collection Form, and included demographic, possible mode of $\mathrm{HCV}$ infection, clinical, biochemical, serological, and virologic data. All patients' data was tabulated and processed using the Statistical Package of Social Sciences (SPSS) version 15.0 for Windows XP (Chicago, IL, USA). The quantitative data was described with mean, median, standard deviation (SD) or range and compared with student's t-test. Pearson correlation was conducted to correlate continuous parameters. Multivariate backwards stepwise binary logistic regression analysis with severe fibrosis $(\mathrm{F} \geq$ $3)$ - as the dependent factor - was performed. To know how well the FIB-5 test compared to FIB-4 as a diagnostic test can predict that a patient has non-severe (F0-2) or severe fibrosis (F3-4), the statistics positive predictive value (PPV), negative predictive value (NPV), sensitivity and specificity were used. Efficiency is an overall estimate of a test's ability to classify patients correctly. It is estimated by adding the numbers of the two correct classifications (true positive and true negative) and dividing by the total number of patients assessed. ROC (receiver operator characterristic) curve(s) were constructed to assess area under the curve (AUROC). Patients were classified into two groups (below and above the cutoff values). Best cutoff values for the independent variables were determined based on the nearest point to top left point in the ROC curve. $\mathrm{P}$ value $<0.05$ was considered significant.

\section{Results}

\subsection{Patient's data}

According to METAVIR system, the determination of liver fibrosis showed stage F0 in 19 (3.1\%), F1 in 372 (61.6\%), 112 $(18.5 \%)$ patients in $\mathrm{F} 2$ and $83(13.7 \%)$ patients were F3 stage and 18 (3.0\%) patients were F4 (Table 2). According to fibrosis stage, all patients were classified into two groups, the first group considered F0-2 as (non-severe fibrosis) and the second group considered F3-4 as (severe fibrosis).

\subsection{Relationship between clinical findings and fibrosis}

Liver fibrosis stages were statistically significant between age groups $(\mathrm{p}=0.000)$. Non-severe fibrosis was diagnosed mostly in younger patients ( $<40$ years), while more advanced stages were observed in patients over 40 years old, table (1). Liver biochemical tests (serum albumin, platelet count, ALT, AST, and ALP) levels were significantly different in various groups in both groups, table (1). ALT and AST 
increased in advanced fibrosis, while ALP, albumin, and platelet count decreased in groups of advanced fibrosis. FIB-5 value and FIB-4 differentiated significantly between fibrosis groups $(\mathrm{p}<0.05)$, table (1). The relationship between fibrosis stages and the two fibrosis scores (FIB-5 and FIB4) is illustrated in figure 1 and tables 2 and 3. There was a significant relationship between fibrosis stages and both serum indexes. A significant increase $(\mathrm{p}=0.000)$ in the level of FIB-4 as fibrosis progresses from non-severe (F0-2) to severe fibrosis (F3-4). A decrease in the level of FIB-5 $(\mathrm{p}=0.000)$ was observed with the progression of fibrosis stages from nonsevere to severe fibrosis, table (1). The AUROCs ( $P$ value) of the serum non- invasive indexes are shown in figure (1-a, b B). AUROCs ( $p$ value) of FIB-5 for differentiating non-severe fibrosis from severe fibrosis (Figure 1:A) was 0.784 $(\mathrm{p}=0.000)$ and for FIB-4 Figure $(1: \mathrm{B})$, it was $0.816(\mathrm{p}=0.000)$. Table 3 depicts the diagnostic performance of FIB-5 and FIB-4 models for the diagnosis of severe fibrosis stages (F0-2) by using the cutoffs (-2.1, $3.25)$ for FIB-5 and FIB-4 respectively. When compared to Liver biopsy, FIB-5 values $\geq-2.1$ showed a NPV of $88.7 \%$ for the diagnosis of non-severe fibrosis (F0-2) with sensitivity of $39.6 \%$. On the other hand, FIB-4 at cutoff $\leq 3.25$ could indicate to non-severe fibrosis (F0-2) with a sensitivity of $86.5 \%$ and a NPV of $87.4 \%$.

Table (1) Baseline characteristics of chronic hepatitis $\mathrm{C}$ patients with hepatic fibrosis regards two main classifications $(n=604)$

\begin{tabular}{|c|c|c|c|}
\hline Variables & $\begin{array}{c}\text { Severe fibrosis } \\
\qquad \begin{array}{c}\text { F3-4) } \\
(n=101)\end{array}\end{array}$ & $\begin{array}{c}\text { Non severe fibrosis } \\
\qquad \begin{array}{c}\text { F0-2) } \\
(\mathbf{n}=\mathbf{5 0 3})\end{array} \\
\end{array}$ & p-value \\
\hline Age (years) & $42.2 \pm 8.3$ & $36.8 \pm 9.6$ & 0.000 \\
\hline ALT & $56(10-435)$ & $42.5(8-239)$ & 0.000 \\
\hline AST & $61(11-264)$ & $40(7-281)$ & 0.000 \\
\hline ALP & $67.5 \pm 26.4$ & $73.5 \pm 21.1$ & 0.013 \\
\hline Albumin & $4.2 \pm 0.4$ & $4.4 \pm 0.3$ & 0.000 \\
\hline Platelet & $155.1 \pm 63.0$ & $226.1 \pm 61.2$ & 0.000 \\
\hline FIB-4 & $2.27(0.28-11.70)$ & $1.01(0.11-8.32)$ & 0.000 \\
\hline FIB-5 & $-1.10(-26-12.13)$ & $3.9(-18.6-17.5)$ & 0.000 \\
\hline
\end{tabular}

ALT; alanine aminotransferase. AST; aspartate aminotransferase. ALP; alkaline phosphatase. Data expressed as mean SD or median (range).

Table (2): Frequency distribution of different biopsy groups.

\begin{tabular}{|c|c|c|}
\hline Stage & Frequency & Percent $(\%)$ \\
\hline F0 & 19 & 3.1 \\
\hline $\mathrm{F} 1$ & 372 & 61.6 \\
\hline $\mathrm{F} 2$ & 112 & 18.5 \\
\hline F3 & 83 & 13.7 \\
\hline F4 & 18 & 3.0 \\
\hline Total & 604 & 100.0 \\
\hline
\end{tabular}

By METAVIR Score 
Table (3) Performance characteristic of both fibro fast and FIB-4 to differentiate between F3, F4 and others fibrosis stages.

\begin{tabular}{cccccccc}
\hline & Sn* & Sp & PPV & NPV & Accuracy & $\begin{array}{c}\text { No. of detected } \\
\text { cases } \\
\text { (\% of all cases) }\end{array}$ & $\begin{array}{c}\text { No. of false positive } \\
\text { cases } \\
\text { (\% of detected cases) }\end{array}$ \\
\hline FIB-5 (-2.1) & $39.6 \%$ & $94.8 \%$ & $60.6 \%$ & $88.7 \%$ & $85.6 \%$ & $40(6.6 \%)$ & $26(39.4 \%)$ \\
FIB-4 (3.25) & $29.7 \%$ & $97.8 \%$ & $73.2 \%$ & $87.4 \%$ & $86.5 \%$ & $30(5 \%)$ & $11(26.8 \%)$ \\
\hline
\end{tabular}

*Sn: sensitivity, SP: specificity, PPV: positive predictive value, $N P V$ : negative predictive value
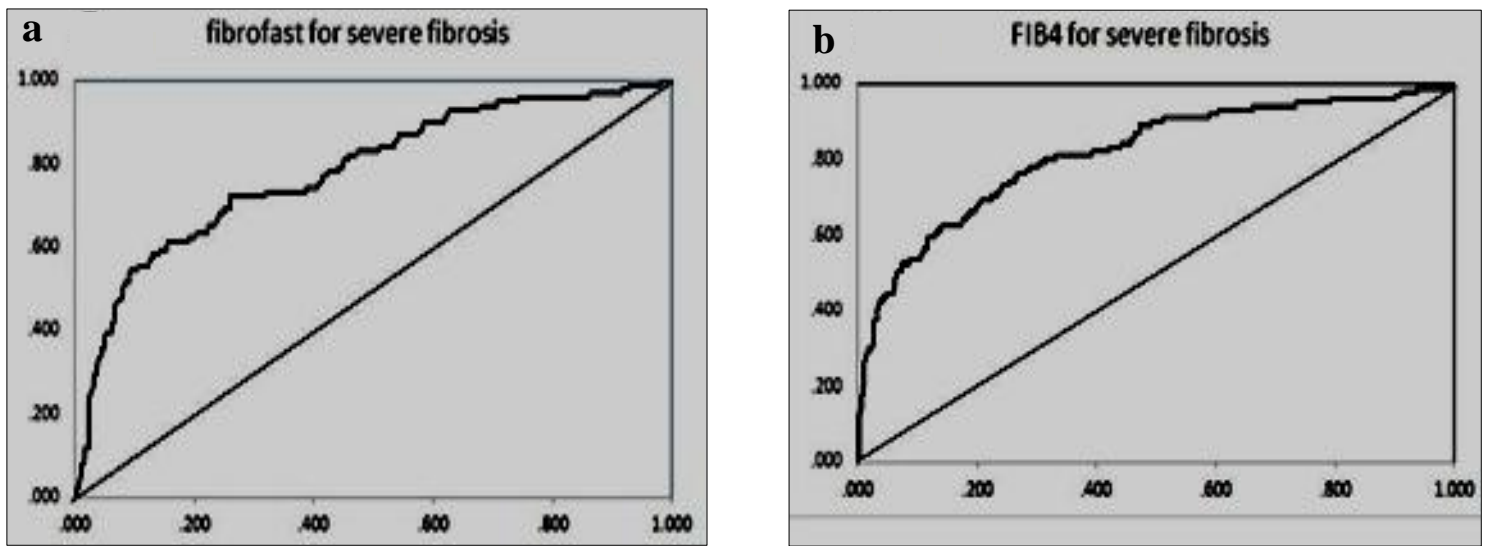

Figure (1) Receiver-operating characteristic curves (ROC) curve generated by FIB-5 (A) and FIB-4 (B) for differentiation between severe and non-severe fibrosis. AUROC curve $=0.816(\mathrm{P}=0.000), 0.784(\mathrm{P}=0.000)$ for FIB-4 and FIB-5, respectively.

\section{Discussion}

For long time, pathological examination of liver puncture tissue was the way to diagnose liver fibrosis. Usage of liver biopsy, because of its invasive trait and sampling errors, is still limited in clinical practice, although it is the gold standard [30,31]. Searching for noninvasive markers to diagnose liver fibrosis has demanded great attention [32-33]. Comparing pathological classification with some noninvasive markers (FIB-5 and FIB-4) to appraise importance of these markers in expressing pathological differences in Egyptian patients with $\mathrm{CHC}$ genotype 4 was the main goal of this study. FIB-5 is a newly adopted score that depends on the combination of five routine laboratory markers (albumin, AST, ALT, alkaline phosphatase, and platelet count) for the detection of hepatic fibrosis in patients with CHC [28]. The FIB-4 is also a noninvasive method for the evaluation of liver fibrosis, based on simple variables such as age, AST, ALT and platelet count. It was initially proposed by researchers of the APRICOT study (AIDS Pegasys Ribavirin International Coinfection Trial) to evaluate the presence of liver fibrosis in HIV/HCV coinfected patients [35] and was subsequently validated in $\mathrm{HCV}$ monoinfected patients [28]. In the present study, the AUROC of FIB-4 to differentiate severe $(\mathrm{F} 3-4, \mathrm{n}=101)$ from non-severe fibrosis ( $\mathrm{F} 0-2, \mathrm{n}=503)$ was 0.816 , with a sensitivity of $29.7 \%$ and a specificity of $97.8 \%$ for cutoff of 3.25 . The NPV was $87.4 \%$ and the PPV was $73.2 \%$ with an accuracy of $86.5 \%$. Similar results were obtained by other authors who evaluated the performance of FIB-4 in $\mathrm{HCV}$ monoinfected patients, with the 
AUROCs ranging between 0.732 and 0.799 [36-40]. When the diagnostic performance of FIB-5 was compared to the FIB-4, FIB-5 was found to be better than FIB-4 for diagnosing non-severe (F02). FIB-5 using the new cutoff value (> 2.1) showed a NPV of $88.7 \%$ for the diagnosis of non-severe fibrosis (F0-2) with sensitivity of $39.6 \%$. According to the results of Attallah et al., 2006 [28] , a cutoff zero on FIB-5 score was previously suggested to be a cutoff point between F02 and F3-4 on METAVIR staging with 98\% sensitivity, 97\% specificity, 99\% PPV, and 92\% NPV. Fenili Amorim et al., 2012 [42] observed an AUROC of FIB-4 to detect significant fibrosis of $0.811 \pm 0.045$, with a sensitivity of $63 \%$ and $28 \%$ and a specificity of $82 \%$ and $99 \%$ for cutoffs of $<1.45$ and $>3.25$ respectively. The NPV was $81 \%$ for FIB-4 values. Vallet Pichard et al [30] observed an AUROC of 0.85 for identifying fibrosis (F3-4), In HCV monoinfected patients. The cutoff point $<1.45$ showed a NPV of $94.7 \%$, with a sensitivity and specificity of $74.3 \%$ and $80.1 \%$ respectively. FIB-4 values 3.25 have a PPV of $82.1 \%$ with lower sensitivity (37.6\%) and higher specificity (98\%). In the study of Elnakeeb et al., 2014 [43], the FIB-4 index proved to be sensitive and specific in the differentiation between patient with no or mild fibrosis (METAVIR F0-1) and patients with significant fibrosis or cirrhosis (F2-4) (AUC=91.6) with the best cutoff value at 1.61 where sensitivity was $69.5 \%$ and specificity was $100 \%$. The PPV was $100 \%$ to detect patient with no or mild fibrosis. Using this cutoff (1.61), $87 \%$ of patients fell outside these ranges and could thus avoid liver biopsy with an overall accuracy of 70\%. Sterling et al., 2006 [36] who proposed the use of FIB-4 index in patients with HIV/HCV coinfection, found that FIB-4 index can differentiate between Ishak stage 0-3 and 4-6. At a cutoff of $<1.45$, the negative predictive value to exclude advanced fibrosis (stage 4-6) was $90 \%$ with a sensitivity of $70 \%$. A cutoff of $>3.25$ had a positive predictive value of $65 \%$ and a specificity of $97 \%$. ValletPichard et al., 2007 [30] evaluated the use of FIB-4 index in 847 patients with $\mathrm{HCV}$ monoinfection, found that FIB-4 index higher than 3.25 had a positive predictive value to confirm the existence of a severe fibrosis (F3-4) of $82.1 \%$ with a specificity of $98.2 \%$. Sumida et al., 2012 [44] evaluated the use of FIB-4 in 576 patients with nonalcoholic fatty liver disease, found that a FIB-4 index higher than 3.25 had a positive predictive value to confirm the existence of a severe fibrosis.

\section{Conclusion}

This study demonstrated that FIB-5 was superior to FIB-4 in the diagnosis of nonsevere fibrosis in patients with chronic hepatitis $c$.

\section{References}

[1] Everhart, J., Wright, E., Goodman, Z., et al., (2010). Prognostic value of Ishak fibrosis stage: findings from the hepatitis $\mathrm{C}$ antiviral long-term treatment against cirrhosis trial. Hepatology 51: 585-594.

[2] Lawson, A., Hagan, S., Rye, K., et al., (2007). The natural history of hepatitis $\mathrm{C}$ with severe hepatic fibrosis. J Hepatol 47: 37-45. 
[3] Ghany, M., Strader, D., Thomas, D., Seeff, L., (2009). Diagnosis, management, and treatment of hepatitis C: an update. Hepatology 49: 1335-1374.

[4] European Association for the Study of the Liver. (2011). EASL Clinical Practice Guidelines: management of hepatitis C virus infection. J Hepatol 55: 245-264.

[5] Rockey, D., Caldwell, S., Goodman, Z., et al., (2009). Liver biopsy. Hepatology 49: 1017-1044.

[6] Regev, A., Berho, M., Jeffers, L., et al., (2002). Sampling error and intraobserver variation in liver biopsy in patients with chronic $\mathrm{HCV}$ infection. Am J Gastroenterol 97: 2614-2618.

[7] Siddique, I., El-Naga, H., Madda, J., et al., (2003). Sampling variability on percutaneous liver biopsy in patients with chronic hepatitis $\mathrm{C}$ virus infection. Scand J Gastroenterol 38: 427-432.

[8] Scheuer, P., (2003). Liver biopsy size matters in chronic hepatitis: bigger is better. Hepatology 38: 1356-1358.

[9] Bedossa, P., Carrat, F., (2009). Liver biopsy: the best, not the gold standard. J Hepatol 50: 1-3.

[10] Poynard, T., Muntenau, M., Morra, R., et al., (2008). Methodological aspects of the interpretation of noninvasive biomarkers of liver fibrosis: a 2008 update. Gastroenterol Clin Biol 32: 8-21.

[11] Bedossa, P., Dargère, D., Paradis, V., (2003). Sampling variability of liver fibrosis in chronic hepatitis $\mathrm{C}$. Hepatology 38: 1449-1457.
[12] Afdhal, N., Nunes, D., (2004). Evaluation of liver fibrosis: a concise review. Am J Gastroenterol 99: 11601174.

[13] Hübscher, S., (1998). Histological grading and staging in chronic hepatitis: clinical applications and problems. J Hepatol 29: 1015-1022.

[14] Schlichting, P., Hølund, B., Poulsen, H., (1983). Liver biopsy in chronic aggressive hepatitis. Diagnostic reproducibility in relation to size of specimen. Scand J Gastroenterol 18: 27-32.

[15] Shiha, G., Sarin, S., Ibrahim, A., et al., (2009). Liver fibrosis: Consensus recommendations of the Asian Pacific Association for the study of the liver (APASL). Hepatol Int 3: 323-333.

[16] Cholongitas, E., Senzolo, M., Standish, R., et al., (2006). A systematic review of the quality of liver biopsy specimens. Am J Clin Pathol 125: 710-721.

[17] Yilmaz, Y., Yonal, O., Kurt, R., et al., (2011). Noninvasive assessment of liver fibrosis with the aspartate transaminase to platelet ratio index (APRI): Usefulness in patients with chronic liver disease. Hepat Mon 11: 103-106.

[18] Leroy, V., (2008). Other non-invasive markers of liver fibrosis. Gastroenterol Clin Biol 32: 52-57.

[19] Pinzani, M., (2006). Non-invasive evaluation of hepatic fibrosis: don't count your chickens before they're hatched. Gut 55: 310-312.

[20] Pohl, A., Behling, C., Oliver, D., et al., (2001). Serum aminotransferase levels and platelet counts as 
predictors of degree of fibrosis in chronic hepatitis $\mathrm{C}$ virus infection. Am J Gastroenterol 96: 3142-3146.

[21] Sebastiani, G., Alberti, A., (2006). Non-invasive fibrosis biomarkers reduce but not substitute the need for liver biopsy. World J Gastroenterol 12: 3682-3694.

[22] Leroy, V., Halfon, P., Bacq, Y., et al., (2008). Diagnostic accuracy, reproduceibility and robustness of fibrosis blood tests in chronic hepatitis C: a meta-analysis with individual data.

Clin Biochem 41: 1368-1376.

[23] Pinzani, M., Vizzutti, F., Arena, U., Marra, F., (2008). Technology Insight: noninvasive assessment of liver fibrosis by biochemical scores and elastography. Nat Clin Pract Gastroenterol Hepatol 5: 95-106.

[24] Lok, A., Ghany, M., Goodman, Z., et al., (2005). Predicting cirrhosis in patients with hepatitis $\mathrm{C}$ based on standard laboratory tests: Results of the HALT-C cohort. Hepatology 42: 282-292.

[25] Silva, Jr., Fakhouri, R., Nascimento, T., et al., (2008). Aspartate aminotransferase- to- platelet ratio index for fibrosis and cirrhosis prediction in chronic hepatitis C patients. Braz $\boldsymbol{J}$ Infect Dis 12: 15-19.

[26] Loaeza-del-Castillo, A., Paz-Pineda, F., Oviedo-Cárdenas, E., et al., (2008). AST to platelet ratio index (APRI) for the noninvasive evaluation of liver fibrosis. Ann Hepatol 7: 350-357.

[27] Degos, F., Perez, P., Roche, B., et al., (2010). Diagnostic accuracy of FibroScan and comparison to liver fibrosis biomarkers in chronic viral hepatitis: a multicenter prospective study (the FIBROSTIC study). $\boldsymbol{J}$ Hepatol 53 (6): 1013-1021.

[28] Attallah, A., Shiha, G., Omran, M., Zalata, K., (2006). A discriminant score based on routine laboratory blood tests for accurate diagnosis of severe fibrosis and/or liver cirrhosis in Egyptian patients with chronic hepatitis C. Hepatol Res Mar; 34 (3): 163-169.

[29] The French METAVIR Cooperative Study Group, (1994). Intraobserver and interobserver variations in liver biopsy interpretation in patients with chronic hepatitis C. Hepatology 1994, 15: 20-20.

[30] Vallet-Pichard, A., Mallet, V., Nalpas, B., et al., (2007). FIB-4: an inexpensive and accurate marker of fibrosis in $\mathrm{HCV}$ infection. Comparison with liver biopsy and Fibro Test. Hepatology 46: 32-36.

[31] Lu, L., Zeng, M., Wan, M., et al., (2003). Grading and staging of hepatic fibrosis, and its relationship with noninvasive diagnostic parameters. World J Gastroenterol 9: 25742578.

[32] Bravo, A., Sheth, S., Chopra, S., (2001). Liver biopsy. N Engl J Med 344: 495-500.

[33] Pilette, C., Rousselet, M., Bedossa, P., et al., (1998). Histopathological evaluation of liver fibrosis: quantitative image analysis vs semi-quantitative scores. Comparison with serum markers. J Hepatol 28: 439-446.

[34] Thabut, D., Simon, M., Myers, R., et al., (2003). Noninvasive prediction of fibrosis in patients with chronic hepatitis C. Hepatology 37: 1220-1221. 
[35] Myers, R., Ratziu, V., Imbert-Bismut, F., et al., (2002). Biochemical markers of liver fibrosis: a comparison with historical features in patients with chronic hepatitis C. Am J Gastroenterol 97: 2419-2425.

[36] Sterling, R., Lissen, E., Clumeck, N., et al., (2006). Development of a simple noninvasive index to predict significant fibrosis in patients with HIV/HCV coinfection. Hepatology 43: 1317-1325.

[37] Zarski, J., Srurm, N., Guechot, J., et al., (2012). Comparison of nine blood tests and transient elastography for liver fibrosis in chronic hepatitis C; The ANRS HCEP-23 study. $\boldsymbol{J}$ Hepatol 56: 55-62.

[38] Adler, M., Gulbis, B., Moreno, C., et al., (2008). The predictive value of Fib-4 versus Fibrotest, APRI, Fibroindex and Forns index to non invasively estimate fibrosis in hepatitis $\mathrm{C}$ and nonhepatitis $\mathrm{C}$ liver diseases. Hepatology 47:762-763.

[39] Cales, P., de Ledinghen, V., Halfon, P., et al., (2008). Evaluating the accuracy and increasing the reliable diagnosis rate of blood tests for liver fibrosis in chronic hepatitis C. Liver Int 28: 1352-1362.
[40] Hsieh, Y.Y., Tung, S.Y., Lee, K., et al., (2012). Routine blood tests to predict liver fibrosis in chronic hepatitis C. World J Gasroenterol 18: 746-753.

[41] Ahmed, W., Ijaz, B., Javed, F.T., et al., (2011). A comparison of four fibrosis indexes in chronic $\mathrm{HCV}$ : Development of a new fibrosiscirrhosis index (FCI). BMC Gastroenterol: 11-44.

[42] Fenili Amorim, T., Staub, G., Lazzarotto, C., et al., (2012). Validation and comparison of simple noninvasive models for the prediction of liver fibrosis in chronic hepatitis C. Ann hepatol 11: 855-861.

[43] El Nakeeb, N., Helmy, A., Saleh, S., et al., (2014). Comparison between FIB-4 Index and Fibroscan as Marker of Fibrosis in Chronic HCV Infection in Egyptian Patients. Open J Gastroenterol 4: 383-391.

[44] Sumida, Y., Yoneda, M., Hyogo, H., et al., (2012). Validation of the FIB-4 Index in a Japanese Nonalcoholic Fatty Liver Disease Population. BMC Gastroenterol;12: 2. 